\title{
Clinical utility of low branched-chain amino acid modular diets in patients with isovaleric aciduria and maple syrup urine disease
}

\section{Suthida Chatvuttinun ${ }^{1}$, Duangrurdee Wattanasirichaigoon ${ }^{2 \star}$, Visith Chavasit $^{3}$, Oraporn Dumrongwongsiri' ${ }^{2}$, Thipwimol Tim-Aroon ${ }^{2}$, Umaporn Suthutvoravut ${ }^{2}$ \& Nalinee Chongviriyaphan ${ }^{2}$}

${ }^{1}$ Graduate Program in Nutrition, Faculty of Medicine Ramathibodi Hospital and Institute of Nutrition, Mahidol University, Bangkok, 10400, Thailand; ${ }^{2}$ Department of Pediatrics, Faculty of Medicine Ramathibodi Hospital, Mahidol University, Bangkok, 10400, Thailand; ${ }^{3}$ Food Science Unit, Institute of Nutrition, Mahidol University, Nakhonpathom, 73170, Thailand

\begin{abstract}
Introduction: Modular diets (MDs) with low amount of offending amino acids have been developed using locally available food ingredients as alternatives to commercial formulas for the treatment of branched-chain organic acidurias (BCOAs). Herein, we conducted a clinical investigation of MDs in patients with BCOAs. Methods: Modular diet A (MDA), with low leucine was produced for maple syrup urine disease (MSUD), and modular diet $\mathrm{B}$ (MDB) products, MDB-1, $-2,-3$, and -4 , with low leucine, valine, methionine and threonine were made for isovaleric aciduria (IVA)/methylmalonic aciduria (MMA)/propionic aciduria (PA). Children aged 4-18 years, with MSUD, IVA, PA or MMA were invited to participate in the study. The research subjects switched from metabolic formula protocol to modular diet protocol. They were followed-up at $0,1,2,4$, and 6 months. Clinical efficacies of MDs were determined by completion of study, compliance to MDs, clinical outcomes and complications, and parental satisfaction. Results: Six children (2 MSUD and 4 IVA) participated and completed the study. Compliance to MDA was $100 \%$ in MSUD subjects with G-tube feeding, while compliance to MDB varied among self-fed individuals with IVA. One subject with MSUD was clinically stable throughout the study, while the other experienced metabolic instability. All IVA individuals showed clinical and laboratory stability during the study. One MSUD and three IVA families preferred the metabolic formula, whereas the other IVA family reported no preference and the other MSUD subject preferred MDs. Conclusion: We provided a proof of concept in developing modular diets for BCOAs, and showed favourable outcomes when using MDs in IVA and varying clinical benefits in MSUD.
\end{abstract}

Keywords: branched-chain organic acidurias, isovaleric aciduria, maple syrup urine disease, metabolic disorders, modular diet

*Corresponding author: Duangrurdee Wattanasirichaigoon

Division of Medical Genetics, Department of Pediatrics, Faculty of Medicine, Ramathibodi Hospital, Mahidol University, Bangkok, 10400, Thailand

Tel: (+66) 22012782; Fax: (+66) 22012783; Email: duangrurdee.wat@mahidol.ac.th

doi: https://doi.org/10.31246/mjn-2020-0071 


\section{INTRODUCTION}

Branched-chain organic acidurias (BCOAs) are a group of disorders resulted from deficiencies of branchedchain amino acids (BCAAs) catabolism enzymes. The most common disorders of this group are maple syrup urine disease (MSUD), isovaleric aciduria (IVA), propionic aciduria (PA), and methylmalonic aciduria (MMA). These disorders are characterized by ketoacidosis and acute/progressive neurological involvement, caused by accumulation of toxic metabolites proximal to the metabolic block (Leonard \& Morris, 2000; de Baulny, Vici \& Wendel, 2012; Saudubray et al., 2002).

During an acute metabolic crisis, dialysis or haemofiltration are often performed in order to remove the toxic metabolites and prevent severe permanent brain damage and death (de Baulny et al., 2012; Saudubray et al., 2002; Zand et al., 2008). For longterm treatment, dietary management is mandatory. The primary goals of dietary management include the promotion of growth and development, prevention of the accumulation of toxic metabolites, enhancement of the excretion of harmful substances accumulated, administration of co-factors, and close monitoring for nutritional deficiencies (Manoli \& Venditti, 2016). Low protein diets in combination with diseasespecific amino acid-free medical formulas, known as metabolic formulas, are recommended to stabilize the metabolic conditions, and support normal growth and neurodevelopment (Knerr et al., 2012; de Baulny et al., 2012; Yannicelli, 2006). The metabolic formulas are manufactured in powder form in which crystalline amino acids voiding of the offending amino acids are used as protein sources. These formulas are enriched with vitamins, minerals, and some with fatty acids, as well as additional sources of energy (Marriage, 2010; Strauss et al., 2010).

Currently, these metabolic formulas are not registered under the Thai Food and Drug Administration (FDA) due to a lack of interest by the companies as a result of small market size and expenses related to the registration process. In addition, the cost of treatment using these metabolic foods is not covered by any health schemes. The metabolic formulas currently used in Thailand have been donated from a single production company. There has been periodic shortage of these medical foods, which contribute to suboptimal treatment in patients. Direct import of these products by the families is problematic due to custom issues and its status as nonregistered medical products. While the availability of medical products and periodic shortage are still unresolved, an alternative measure needs to be developed for temporary resolution.

Modular diets (MDs) contain single or multiple nutrients which are combined to fulfil a specified need in medical treatment (Davis \& Baker, 1996). The use of MDs allows flexible mixing ratio; therefore, one food ingredient module can be combined with another. Food ingredients are available in general markets, over the counter or by prescription. Examples of carbohydrate modules are starch, polysaccharides, glucose polymers, mono- and disaccharides. Mediumchained triglycerides (MCT) and longchained triglycerides (LCT) oil can be used as fat sources (Davis \& Baker, 1996).

We previously published an article on the development of food products using fish maw and roasted sunflower kernel for patients with branched-chain organic aciduria (Chatvuttinun et al., 2018). Five products were successfully developed: Formula I, low leucine diet for MSUD 
and Formulas II-V, low leucine and valine diet made for IVA, PA, and MMA. Herein, we conducted a clinical study of MDs in children with branched-chain organic acidurias using these previously developed food products, and evaluated their clinical efficacies.

\section{MATERIALS AND METHODS}

Subject recruitment and study design Eligible subjects were children with MSUD, IVA, PA, or MMA, aged 4-18 years who were followed-up at Pediatric Outpatient Clinic, Ramathibodi Hospital. Exclusion criteria included (i) in serious metabolic illness; (ii) have cardiac or kidney disease related to the underlying metabolic disorder; (iii) have a history of multiple $(>2)$ hospitalisations related to their metabolic disorder during one year prior to entering the study; and (iv) subject withdrawal. Parents of the research subjects were required to provide written informed consents. The study was conducted following an approval from the Ramathibodi Hospital Institutional Review Board (ID 09-57-25:MURA2014/ 492S1; MURA2020/1058).

This study was an outpatient-based, single-arm trial lasting a period of six months (March-August 2015). Patients' demographic data including age, sex, underlying diseases and severity, onset of diseases, feeding history, current clinical status, and baseline dietary treatments were reviewed.

\section{Preparation of modular diets (MDs)}

For the convenience of monitoring, we reassigned the MDs in this study into two major formulas - MDA for MSUD and MDB for IVA, PA and MMA. The MDA (previously named as Formula I) contained low leucine, whereas the MDB contained low leucine, valine, isoleucine, methionine, and threonine. The MDs were produced using fried fish maw (Tawan Fish Maw, Tawan Produce
Co., Ltd., Samutprakarn, Thailand) and roasted sunflower kernels (Flower Food Ltd., Bangkok, Thailand), following the established protocols (Chatvuttinun et al., 2018). These food items were selected because they contained minimum/ low ratio of leucine to protein and were produced under systematic farming in which the quality could be controlled and natural variation was minimal. The fish maw was obtained from a farmed fish called "Pla Swai" (Pangasius hypophthalmus), while sunflower kernel (Helianthus annuus) was harvested from a sunflower farm, followed by sorting and roasting. Both raw materials were preserved in dried/roasted and dried/ fried forms, which made them available all year round. These products are commercially and widely available worldwide. The production of MDs was carried out at a facility available at the Institute of Nutrition, Mahidol University.

Given that all eligible MSUD subjects were on G-tube, only mixed powder form of MDA was prepared. For those selffed IVA/MMA/PA patients, four types of MDB products were made available for the patients to choose, namely MDB-1: rice sprinkle powder, MDB-2: bouillon cube for stir-frying, MDB-3: instant cocoa drink, and MDB-4: chocolate snack bar (Figure 1A). The ingredients, nutrient composition, and branchedchain amino acid profiles of MDA and the four MDBs were described in an earlier publication. The MDB-1, MDB2, MDB-3, MDB-4 were formerly named as Formulas II, III, IV, V, respectively (Chatvuttinun et al., 2018).

We informally performed a simple sensory testing among four adult volunteers and three eligible research subjects by giving them a bite try of modular products, then modified the recipes to improve their texture, taste, and flavour as needed. After achieving 
the final recipes, we performed quality analysis of all products including macronutrients, amino acid profiles, and water activity. The MDs produced were also proven to be void of chemical toxicity and were microbiologically safe for food grade. Each product provided 500-600 kcal per $100 \mathrm{~g}$ edible portion. Energy sources of the MDs were mainly from carbohydrate and fat, similar to the metabolic formulas (BCAD2/LMD). However, MDA and MDB as compared to metabolic formulas, contained higher distributive energy from fat $(46-67 \%$ vs. $18-46 \%$ ), and less distributive energy from carbohydrate (24-46\% vs. 41-56\%). Energy distribution from protein source was lower in the MDs as compared to metabolic formulas at $5.8-86 \%$ vs. 13 23\% (Chatvuttinun et al., 2018).

We then calculated energy requirement for each patient, following the HollidaySegar equation and standard nutritional recommendations for severe MSUD, IVA, MMA, and PA (Barshop, 2006; Wappner $\&$ Gibson, 2006). Baseline nutritional intake of each patient was taken into account for preparation of MD-nutrients to be provided. Total protein intake for patients aged 4-8 and 8-15 years were $1.5-2.0$ and $1-1.2 \mathrm{~g} / \mathrm{kg} /$ day, respectively (Wappner \& Gibson, 2006). For MSUD and IVA, leucine was selected as the primary offending amino acid for the calculation of amino acid allowance per day. The daily leucine allowance was 500-750 mg/day for MSUD and $650-1,500 \mathrm{mg} /$ day for IVA because leucine tolerance is known to be higher in individuals with IVA than MSUD (Marriage, 2010; Wappner \& Gibson, 2006). As for MMA and PA patients, valine was chosen as the primary for calculation of maximum daily allowance of offending amino acids, leading to the following assumptions - 700-1600 mg of valine, $600-1300 \mathrm{mg}$ of isoleucine, 500-1200 mg of threonine, and 250-800 $\mathrm{mg}$ of methionine. Each MD product was packaged into a cling wrapped foil sachet to suit individual nutritional requirement. Upon completion of the packaging, microbial contamination was analysed following published protocols (Chatvuttinun et al., 2018).

\section{Metabolic formula protocol, modular diet protocol, and prescription of modular diets}

Metabolic formula (MF) protocol involved medical formula plus "baseline-low protein diet" (baseline-LPD) that the subjects regularly consumed prior to study entry. During the study, subjects were asked to switch from MF-protocol to MD-protocol, which included MDs plus adjusted type and amount of natural low protein diet (adjusted-LPD) following the recommendations by researchers. The amounts of MDs and adjusted-LPD to be consumed per day were calculated for each subject, based on individual body weight, weight-for-height, and baseline amino acid tolerance according to the widely used standard treatment for amino acid disorders. As cooked rice is a basic diet for most Thai people, we started by calculating energy, protein, and leucine intakes from the amount of cooked rice the participating subjects usually consumed and negotiated this to a realistic amount acceptable for them in order to keep the amount of offending amino acids from exceeding the maximum allowance.

The study subjects were asked to continue their routines including school activities. They were asked to visit the research team at $0,1,2,4$, and 6 months for clinical and dietary assessments, checking of compliance to MDs, and for blood tests. A 1 to 2-month supply of MDs was provided to the subjects at each clinic visit. Telephone communication was used as extra follow-ups when necessary. Individuals with IVA were invited to choose specific products, MDB-1 and MDB-2, during 
the first month of the study. In order to provide new choices and to motivate the children's appetite, MDB-3 and MDB-4 were offered as alternatives at the end of the first month and during following visits.

\section{Dietary assessment and compliance monitoring}

Parents were asked to record the amount of MDs and adjusted-LPD consumed by their children daily. Additionally, the parents prepared a three-consecutive day food record prior to each clinic visit. They were required to bring the left-over MDs to the clinic, which allowed the researchers to assess and confirm individual compliance to MDs. Compliance to MDs was defined as percentage of the amount of MDs consumed as compared to the amount prescribed.

\section{Clinical assessment}

At every clinic visit, anthropometric characteristics including weight, height, head circumference, mid arm circumference, and triceps and biceps skinfolds were measured following standard methods. Data on weight, height, length were analysed and expressed as weight-for-age $z$-scores (WAZ), height (length)-for-age $z$-scores (HAZ), and weight-for-height (length) z-scores (WHZ), using the WHO (2006) for the growth standards (for 0-5 years old) and WHO (2007) for the growth reference (for children 5 years and above) (de Onis et al., 2007), and WHO AnthroPlus software version 1.0.4 (available at http: / / www.who.int / growthref / tools/en/). WAZ or HAZ <-2 indicated underweight or stunting, respectively. Plasma amino acid profiles (fasting), electrolytes (including $\mathrm{TCO}_{2}$ content), ammonia, albumin, pre-albumin, blood urea nitrogen, and complete blood count were investigated.
To monitor for metabolic illness and patient safety, information on adverse clinical symptoms, hospital admission, dietary adjustment, and other additional treatments were recorded. Standard sick day and/or emergency protocols were activated when there was medical illness or metabolic crisis (Wappner \& Gipson, 2006). The sick day protocols included home-based close monitoring, reduction of intact protein by $50-100 \%$ for $24-$ 48 hours, increase of fluid hydration, and adding non-protein energy sources such as fruit juices (Wappner \& Gipson, 2006). Once the acute illness/crisis was over, MD-protocol was resumed. If the patient's metabolic condition became uncontrolled, defined as having $\geq 2$ hospitalisations during the study period, the MD-protocol would be terminated for that particular individual. In addition, the subjects continued taking vitamin and mineral supplements at the same dosages that they have received prior to study entry.

\section{Analysis of clinical utility}

Clinical utility of MDs was determined using descriptive analysis, by (i) complete vs. drop-out from the study; (ii) compliance to MDs; (iii) clinical outcomes and complications as evidenced by nutritional status, clinical and metabolic instability, hospitalisations, and adverse laboratory changes; and (iv) parental satisfaction. Parents of subjects were asked, at the end of the study, to express their overall preference on MD-protocol vs. MF-protocol (if they preferred continuation of MD-protocol or MF-protocol). Statistical analysis was not employed due to small number of research subjects.

\section{RESULTS}

\section{Patient characteristics and MD} prescription

There were nine eligible subjects 
including one MMA, three MSUD and five IVA patients. Two patients (MSUD and IVA) declined to take part in the study because long distance travelling and frequent clinic visits were required. The MMA patient was excluded due to having complicated chronic kidney disease. As a result, a total of six subjects including two MSUD and four IVA patients participated in the study. Their age ranged between 4.0-17.3 years. All research subjects had severe neonatal onset of the diseases (Table 1).

Energy, nutrient, and BCAA intakes at baseline and during the study are shown in Table 1. Each individual was equally prescribed to obtain $490 \mathrm{kcal} /$ day from three meals of adjusted-LPDs and the remaining daily calories from MDs at all visits until the end of the study. The caloric intake from MDs ranged $52-66 \%$ of their daily prescribed calories.

\section{Drop-out, compliance to modular diets, and product acceptability}

All participating subjects completed the study with no drop-outs. Compliance to MDA product was $100 \%$ in MSUD subjects. As for IVA self-fed subjects, after one month (M1) of trial, the compliance to MDB-1 and MDB-2 ranged $0-70 \%$ and $20-60 \%$, respectively. With the additional choice of MDB-3, the compliance of most subjects increased to $45-90 \%$ at the end of the second month (M2) and subsequently to $40-100 \%$ during the fourth and sixth months (M4 and M6) of the study (Figure 1B).

\section{Clinical outcomes and complications}

All IVA subjects reported that they were able to continue their daily activities as usual during the study, including attending school and play, whereas those with MSUD (with severe psychomotor delay) stayed at home as

Table 1. Demographic data of the study patients

\begin{tabular}{lcccccc}
\hline Characteristic & Pt. 1 & Pt. 2 & Pt. 3 & Pt. 4 & Pt. 5 & Pt. 6 \\
\hline Underlying disorder & MSUD & MSUD & IVA & IVA & IVA & IVA \\
Sex & male & female & male & male & male & female \\
Age of onset & $5 \mathrm{~d}$. & $7 \mathrm{~d}$. & $11 \mathrm{~d}$. & $3 \mathrm{~d}$. & $8 \mathrm{~d}$. & $4 \mathrm{~d}$. \\
Age at diagnosis & $2 \mathrm{~m}$. & $2 \mathrm{~m}$. & $14 \mathrm{~d}$. & $10 \mathrm{~d}$. & $4 \mathrm{~m}$. & $6 \mathrm{~d}$. \\
Age at entry (years) & 17.3 & 4.4 & 4.6 & 9.7 & 9.0 & 4.0 \\
Current clinical status & & & & & & \\
Psychomotor delay & severe & severe & no & mild & mild & mild \\
Other manifestation & seizure & seizure & ADHD & ADHD & ADHD & no \\
Baseline daily dietary intake & & & & & & \\
Route of delivery & G-tube & G-tube & oral & oral & oral & oral \\
Diet & BCAD2 & BCAD2 & LMD & LMD & LMD & LMD \& \\
& $\&$ LPD & $\&$ LPD & $\&$ LPD & $\&$ LPD & $\&$ LPD & LPD \\
Calories (kcal) & 1819 & 1355 & 1197 & 953 & 991 & 878 \\
Protein (g) & 51.1 & 22.8 & 25.8 & 24.2 & 30.8 & 28.2 \\
Leucine (mg) & 1374 & 735 & 1191 & 1100 & 1804 & 1200 \\
Leucine (mg/kg) & 34.4 & 49.0 & 59.3 & 44.9 & 50.8 & 86.3 \\
\hline
\end{tabular}

Notes: ADHD, attention deficit and hyperactivity disorder; G-tube, gastrostomy tube; IVA, isovaleric acidemia; LPD, low protein diet; MSUD, maple syrup urine disease 
they did previously. During the study, all but one subject (P2), reported no vomiting, gastrointestinal discomfort, or any illnesses. One patient (P1) became neurologically more alert, energetic, and communicative as compared to his baseline. Subject P2, at 2.5 months of the study, developed frequent vomiting whilst having negative urine ketone, normal blood sugar and electrolytes profiles $\left(\mathrm{TCO}_{2} 20\right.$, anion gap 10). This patient was managed at home, following the sick day protocols, with extra juices and calories, which made the symptoms subside within 48 hours. At 4.5 months of the study, the patient had episodic vomiting with hypokalemia and mild metabolic acidosis $\left(\mathrm{K} 2.9, \mathrm{TCO}_{2} 12\right.$, anion gap 17) without ketonuria, necessitating hospitalisation for fluid rehydration for 48 hours. An additional 230 calories per day $(300 \mathrm{~mL}$ of orange juice, apple, and banana) was provided to this patient, yielding no further adverse events during the rest of the study.

Figure $1 \mathrm{C}$ shows that in all subjects but one (P2), the comparison of WAZ between pre- and post-study entry were: normal in three subjects (P3: Z-score of $1.1 / 1.2$ for pre- and post-study; P4:1.6/-1 and P6: $-0.2 /-0.4)$; remained underweight in one (P1: $Z$-score of -3.05/-3.12); remained overweight in the other (P5: Z-score of 1.6/1.8). The WAZ of P2 was negatively changed from normal to underweight $(Z$-score $-0.8 /$ -
$\mathbf{A}$
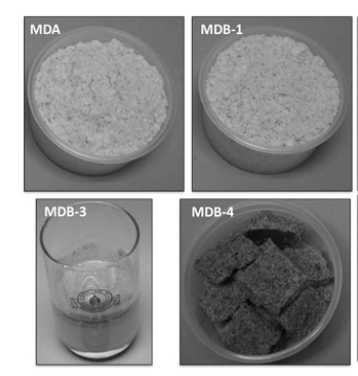

WAZ

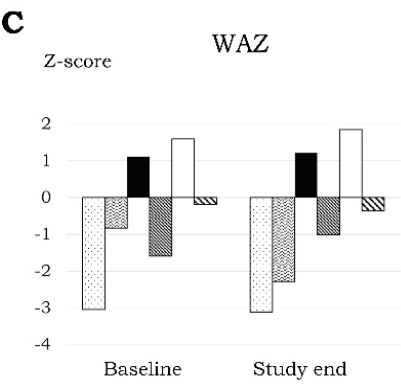

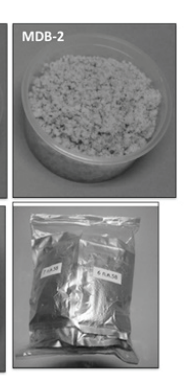

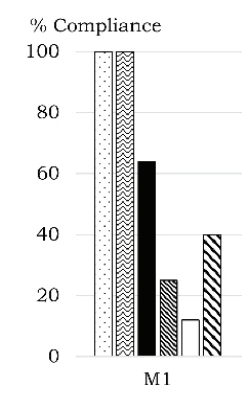

HAZ

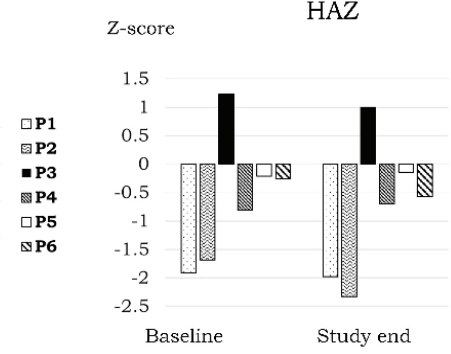

B

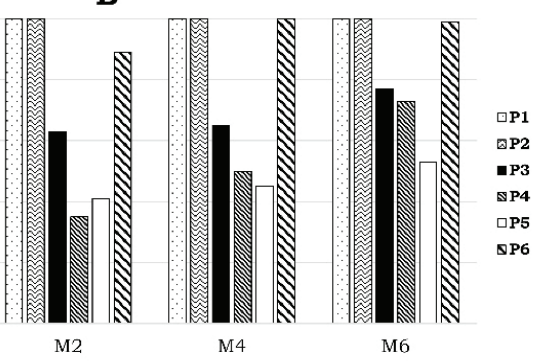

WHZ

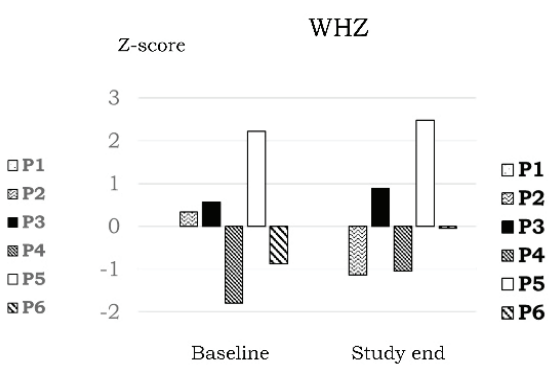

Figure 1. Modular diets, Compliance, and Growth outcomes. A) Five MD products including MDA, mixed powder for MSUD; MDB-1: rice sprinkle powder, MDB-2: bouillon cube for stirfrying, MDB-3: instant cocoa drink, and MDB-4: chocolate snack bar for IVA/PA/MMA. Each MD product was prepared and packaged into clean wrapped foil sachets. B) Compliance to modular diets shown in percentage of the amount of MD consumed as compared to the amount prescribed. C) Growth outcomes. Noted growth changes of the patients as expressed by weight-for-age $z$-scores (WAZ), height (length)-for-age $z$-scores (HAZ), and weight-forheight (length) Z-scores (WHZ). M (month) represents time point: MO, the start of the study; M1, M2, M4 and M6 represent the end of the $1^{\text {st }}, 2^{\text {nd }}, 4^{\text {th }}$ and $6^{\text {th }}$ month of the study, in order. P1-6 represent patients 1-6 in respective order. 
2.2). HAZ remained unaffected in all, but one (P2), as follows: P1: Z-score of $-1.9 /-2.0$ for pre- and post-study; P2: $-1.8 /-2.3$; P3 1.2/1; P4: -0.9/-0.7; P5: $-0.2 /-0.1$; and P6: -0.2/-0.6). As for WHZ, it was unaltered for all subjects: normal in four individuals (P2: $0.3 /-1.1$; P3:0.6/0.9; P4: -1.8/-1.1 and P6: -0.9/0.1 ); overweight in another (P5: 2.1/2.5; Figure 1C). Mid arm circumference, triceps and biceps skinfolds increased in all subjects as predicted for well growing children (data not shown).

Haematocrit, serum $\mathrm{TCO}_{2}$ content, albumin, and pre-albumin were within normal ranges throughout the study (Figure 2). Subjects with MSUD showed low blood urea nitrogen (BUN) values
( $<7 \mathrm{mg} / \mathrm{dL}$ ) prior to and throughout the study, probably due to more restricted protein intake, as compared to normal BUN levels among IVA individuals. Plasma leucine level was periodically within the acceptable range in one MSUD subject (P1), but persistently high in the other (P2; Figure 3). All IVA subjects had normal plasma amino acid profiles before and during the study (Figure 4).

\section{Parental satisfaction}

Three out of four IVA families (P3, P4 and P5) reported preferences for the MF-protocol as the protocol provided flexibility for their children to consume more natural foods with intact protein at school, in addition to drinking LMD
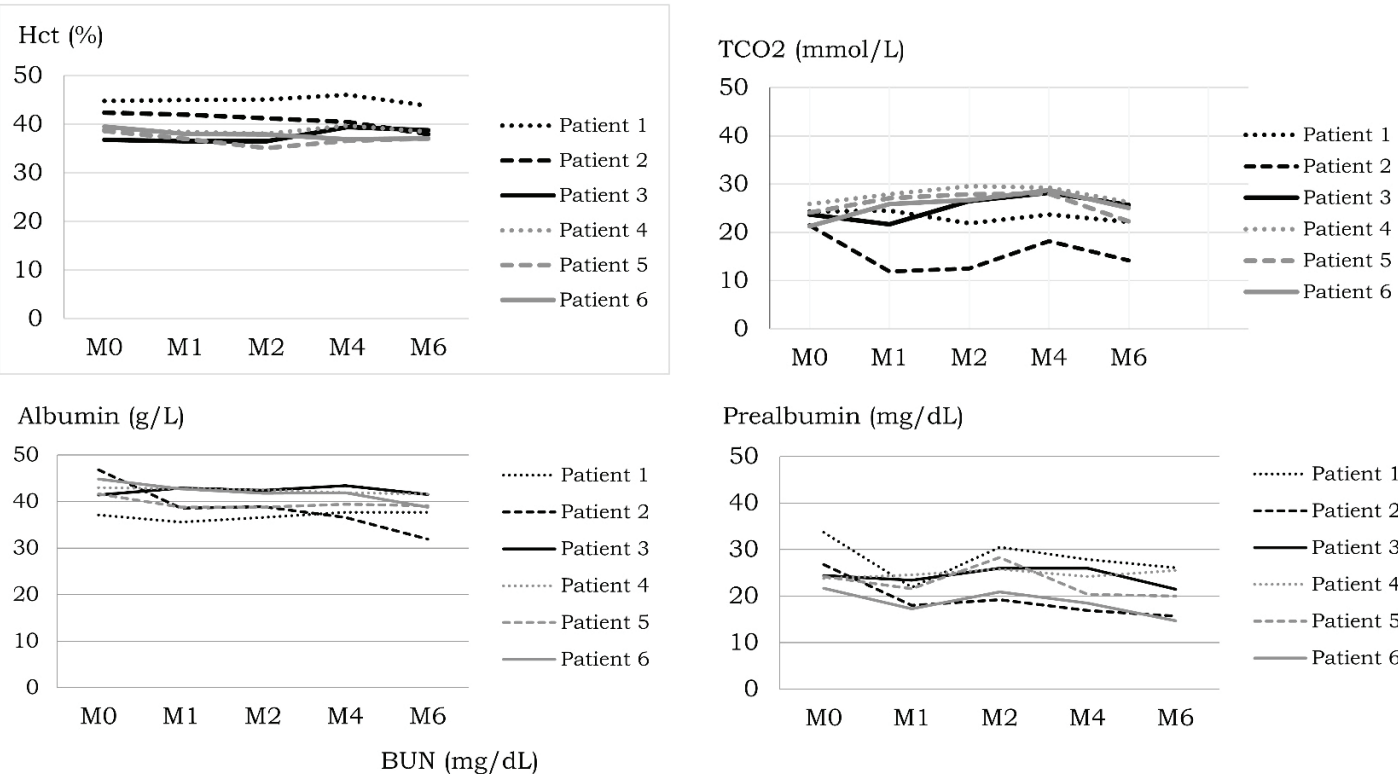

Prealbumin $(\mathrm{mg} / \mathrm{dL})$
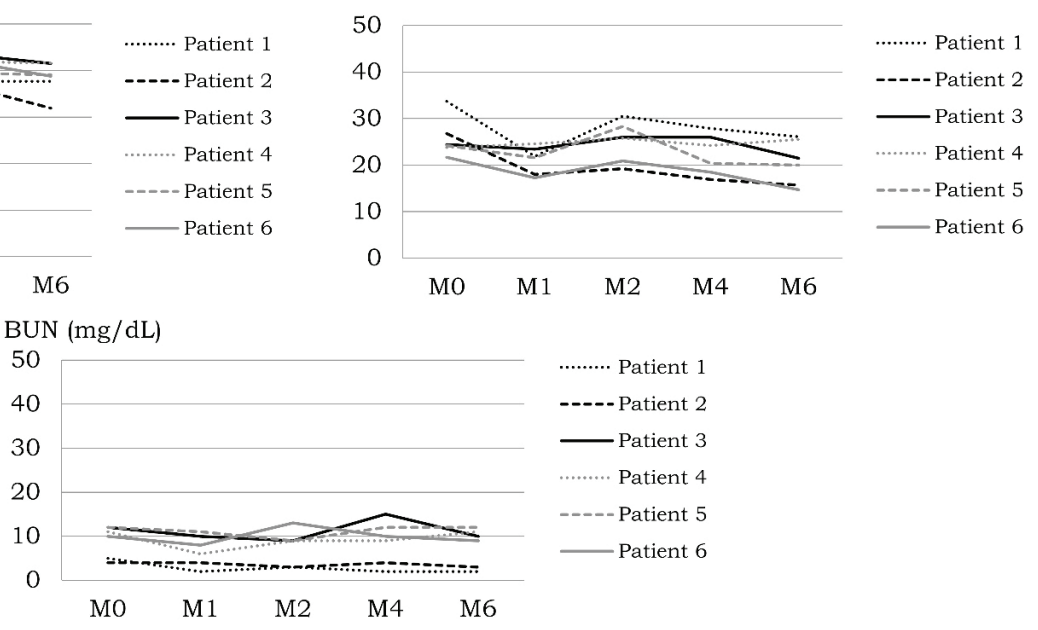

Figure 2. Haematological and biochemical parameters of the patients receiving modular diets. $\mathrm{M}$ (month) represents time point: M0, the start of the study; M1, M2, M4 and M6 represent the end of the $1^{\text {st }}, 2^{\text {nd }}, 4^{\text {th }}$ and $6^{\text {th }}$ month of the study, in order. 
milk. The other IVA family (P6) reported no preference to either diet protocols. One MSUD family (P1) preferred the MD-protocol because their child appeared healthier and more energetic, as compared to baseline treatment. The other MSUD family (P2) preferred MFprotocol because their child was more clinically stable with no illness/weight loss whilst on MF-protocol.

\section{DISCUSSION}

We demonstrated the clinical utility of MDs, using locally available raw materials, namely dry fish maw and roasted sunflower kernels, in patients with MSUD and IVA. The distributive energy of MDs developed was higher from fat and less from carbohydrate, compared to MF. It was not our intention to increase fat intake for caloric requirement, but to maintain the texture and shape of the products. Therefore, we suggest partial replacement of fat module with carbohydrate (dextrin) module in the future. Protein quality of the MDs in this study was considered as "good", based on the standard definition of amino acid scores. "Good protein quality" is achieved when the percentage

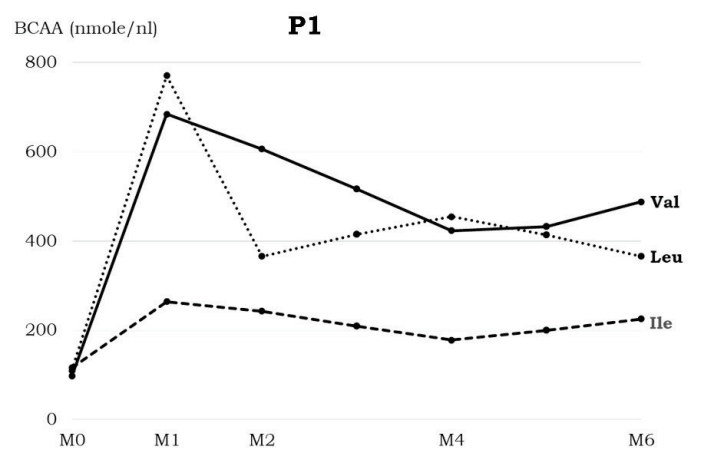

of each essential amino acid consumed by each individual is $\geq 70 \%$ of the recommended amount.

The raw materials, namely dried fish maw and sunflower kernels, are widely available at supermarkets and on E-commerce websites. The easiest products to be made are mixed powder product or rice sprinkle powder (MDA and MDB-1), followed by bouillon cubes for stir-frying (MDB-2), and instant cocoa drink (MDB-3). These products can be simply cooked at home using a regular blender, following the established recipes. The chocolate snack bar (MDB4) was the most difficult to prepare at home because of its time-consuming feature and difficulty in mixing the baking chocolate to other ingredients. To avoid complicated microbial testing steps and its related expenses, the recipes for these MDs need to be given to the families so that they can prepare the MDs correctly at home, on a daily basis.

For self-fed children, product acceptability was a major concern especially when they were forced to switch from their routine diet (metabolic formula) to new products. Therefore, the new products must be competitive in

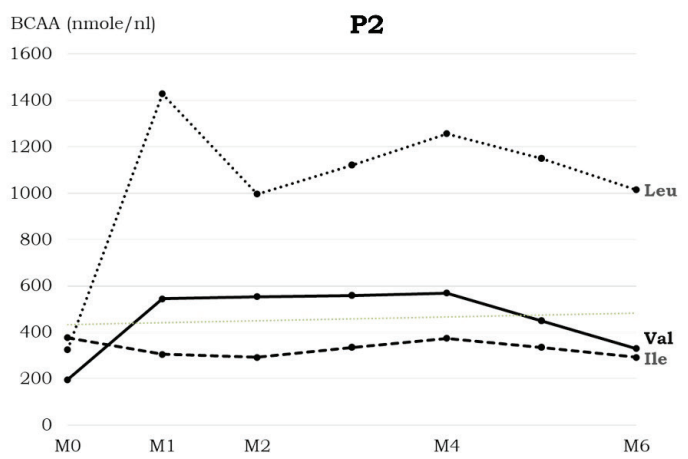

Figure 3. Branched-chain amino acids (BCAA) levels of patients with MSUD. Goal levels of BAAA in MSUD are: Leu and Ile 150-300 $\mu \mathrm{mol} / \mathrm{L}$ (or nmole/ml), and Val at least two-fold plasma leucine concentration. M (month) represents time point: M0, the start of the study; M1, M2, M4 and M6 represent the end of the $1^{\text {st }}, 2^{\text {nd }}, 4^{\text {th }}$ and $6^{\text {th }}$ month of the study, in order. 
terms of texture, flavour, and taste. In this study, the compliance to MDs was quite low during the first month of the trial, but subsequently improved when patients became more familiar with the MD products. We observed that MDB3: instant cocoa drink was the most acceptable among all self-fed children, with $100 \%$ compliance in three out of four patients at the $4^{\text {th }}$ month of the study (Figure 1B). The reason for this is probably due to its liquid texture that is similar to a drinkable metabolic formula. As for chocolate snack bar, two out of four self-fed patients preferred the snack bar to the instant cocoa drink, whereas another showed no preference, and the other totally declined the snack bar. Anecdotally, we found that
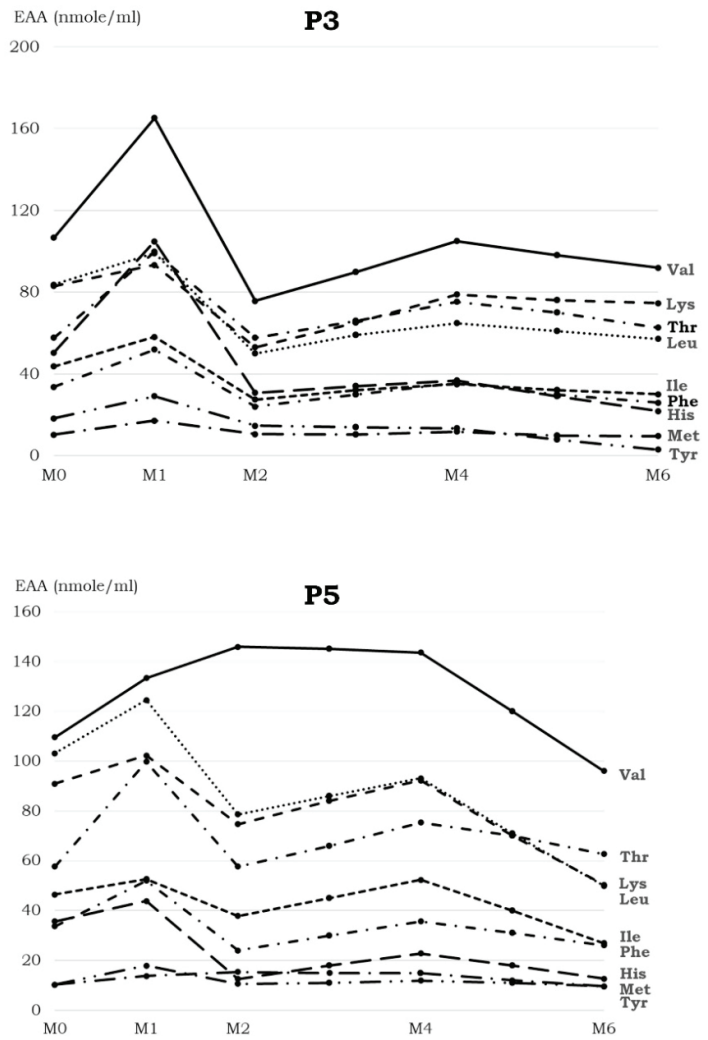

strong encouragement by parents was another key success factor to increase compliance to these MDs.

Clinical outcomes of MDB in IVA were highly favourable since there were no adverse events throughout the study. Despite incomplete compliance to MDs by some IVA subjects, all of them showed normal or unaltered growth parameters, haematologic and biochemical profiles, possibly due to the wide range of safety margin of leucine intake in IVA. Metabolic ketoacidosis in children with IVA often occurs during infancy and early childhood, but the episodes usually decrease with age (Castorina et al., 2008; Dercksen et al., 2012; Martin-Herhandez et al., 2009). This may be one of the reasons that we
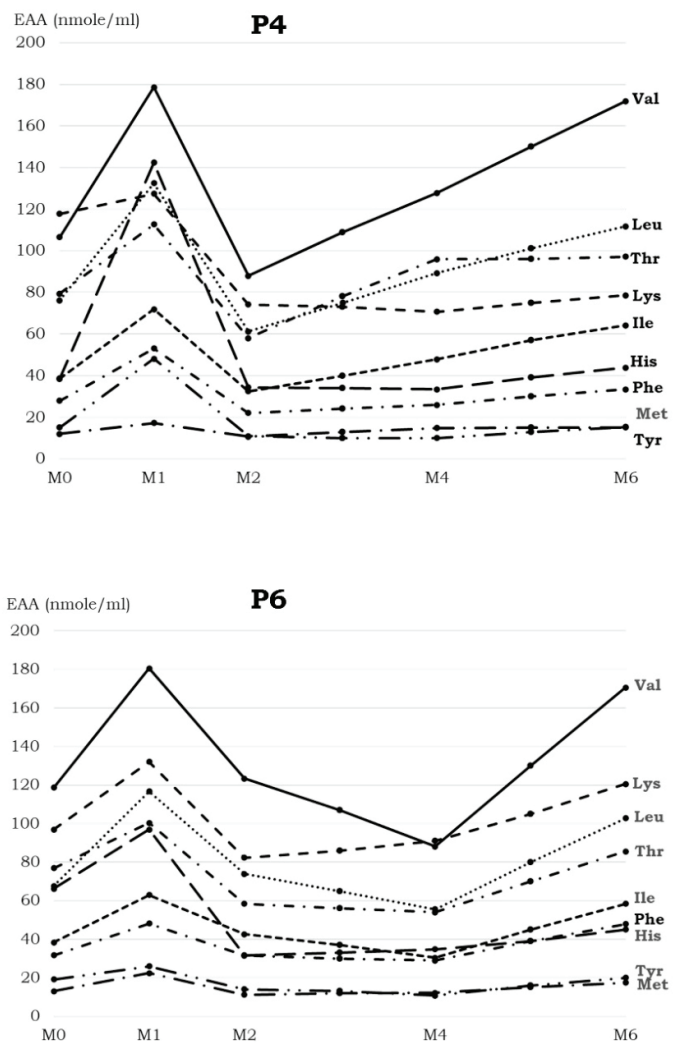

Figure 4. Plasma levels of essential amino acids (EAA) of patients with IVA. Normal range for plasma amino acids are: Val 88-275; Leu 52-149; Ile 25-76; Lys 56-168; Thr 12-138; Phe 41-84; His 8-43; Met 7-42; Tyr 5-24 nmole/ml 
did not see metabolic attack in our IVA children who did not adhere to dietary protocols during the study. Clinical variation of IVA with the same mutation in a homogeneous population has been demonstrated, suggesting that multiple factors, such as poor diet compliance, delayed diagnosis and treatment, epigenetic factors, and yet unidentified factors, may be involved in the variability of manifestations (Dercksen et al., 2012). It has been suggested that there is no reliable metabolomic markers for monitoring the prescribed treatment in patients with IVA during the time of well-being (Vockley \& Ensenauer, 2006). Unsatisfactory diet compliance in IVA could lead to chronic intoxication of abnormal organic acid accumulation, leading to psychomotor delay and neurobehavioral problems including attention deficit, learning disability, and intellectual disability (Castorina et al., 2008).

As for MSUD, the MD-protocol yielded impressive clinical outcomes in one patient (P1) but was unsuccessful or partially effective in the other (P2). MSUD is generally considered more severe and much more difficult to control and therefore requires more stringent dietary management than IVA. The adverse outcomes in this subject (P2) were likely due to several factors including (i) relatively lower caloric and protein intakes during the study; (ii) poorer absorption of amino acids from natural foods (MDs) than from metabolic formulas; and (iii) lower bioavailability of MDs relative to metabolic formulas.

We did not include children aged under 4 years in the study because younger children with these disorders are more vulnerable to metabolic decompensation. Given the impressive results of MDBs for IVA, they may be considered as an alternative in the management of younger IVA patients, if necessary. Theoretically, the MDBs developed in this study can be used for MMA and PA as the amounts of valine, isoleucine, threonine, and methionine calculated were within the allowable ranges. However, further clinical investigation is required.

The development of MDs to be used as substitutes for semi-elemental products and MF have been described, with varying degree of success depending on the conditions. Perhaps the most successful examples are MDs for chronic diarrhoea and malabsorption (Kolacek et al., 1996), hepatic and renal failure (Bell et al., 1987), and burn (Bell \& Wyatt, 1986). The main reason of the development of a MD for the aforementioned disorders was the very high cost of commercial formulas. There has been scarce data on using MDs for organic acidurias, as Kabra reported no clinical success in using modified Indian diet for organic aciduria patients (Kabra, 2002). Although our MDs are not perfect substitutes for metabolic formulas, they can however be used as alternatives during severe shortage and for geographical areas lacking metabolic formulas.

The advantages of the MDs developed in this study included local availability, good protein quality, simple preparation, proven food safety, low cost, and proven clinical efficacy (for IVA). Limitations of this study were small sample size, incomplete compliance to MDs, incomplete metabolomic data such as plasma essential fatty acids, and absence of micronutrient data (vitamins and minerals).

\section{CONCLUSION}

We have provided a proof of concept in developing modular diets for BCOAs using locally available raw materials with low cost and simple preparation, with favourable outcomes in clinical utility among IVA and varying clinical 
benefits in MSUD. A large cohort of clinical study using the developed modular diets in patients with organic acidurias is required. A modular diet can be used as an alternative during the long-time shortage of metabolic formulas in resource-limited communities.

\section{Acknowledgements}

We are thankful to the patients' and their families for participating in the study. This work was funded by the Research Assistant Scholarship from the Faculty of Graduate Studies, Mahidol University, Academic Year 2012 and grant from the Faculty of Medicine, Ramathibodi Hospital (directed to DW). We thank the Faculty of Medicine Ramathibodi Hospital, Mahidol University for promoting the staff on research tract by giving a special award of Research Career Development to DW and TT.

\section{Authors' contributions}

$\mathrm{SC}$, conducted the study and wrote the manuscript draft; DW, involved in the conceptualization and design of the study, supervised data analysis, and wrote the manuscript; VC, supervised modular diet formation and production; OD and TT, provided patient care and clinical data; US and NC, involved in study design and advice on patient care.

\section{Conflict of interest}

The authors declare no conflict of interest.

\section{References}

Barshop B (2006). Disorders of valine-isoleucine metabolism. In N Blau, G Hoffmann, J Leonard and J Clarke (eds). Physician's guide to the treatment and follow-up of metabolic diseases (pp. 81-92) Springer-Verlag, Heidelberg.

Bell SJ, Bistrian BR, Wade JE \& Blackburn GL (1987). Modular enteral diets: cost and nutritional value comparisons. J Am Diet Assoc 87:1526-1530.

Bell SJ \& Wyatt J (1986). Nutrition guidelines for burned patients. J Am Diet Assoc 86:648-653.

Castorina M, Rigante D, Antuzzi D, Sciascia Cannizzaro G \& Ricci R (2008). Different outcome in isovaleric acidemia might be related to unsatisfactory diet compliance. Scand $J$ Gastroenterol 43:767-768.

Chatvuttinun S, Chavasit V, Wattanasirichaigoon D, Suthutvoravut U \& Chongviriyaphan N (2018). Development of food products using fish maw (Pangasius hypophthalmus) and roasted sunflower kernel (Helianthus annuus) for branched-chain organic acidurias patients. Mal J Nutr 24:607-615.
Davis A \& Baker S (1996). The use of modular nutrients in pediatrics. JPEN J Parenter Enteral Nutr 20:228-236.

de Baulny H, Vici C \& Wendel U (2012). Branchedchain organic acidurias/acidaemias. in $\mathrm{J}$ Saudubray, G Berghe and J Walter (eds). Inborn metabolic disease (pp. 278-296) SpringerVerlag, Berlin.

de Onis M, Onyango AW, Borghi E, Siyam A, Nishida C \& Siekmann J (2007). Development of a WHO growth reference for school-aged children and adolescents. Bull World Health Organ 85:660-667

Dercksen M, Duran M, Ijlst L, Mienie LJ, Reinecke CJ, Ruiter JP, Waterham HR \& Wanders RJ (2012). Clinical variability of isovaleric acidemia in a genetically homogeneous population. $J$ Inherit Metab Dis 35:1021-1029.

Kabra M (2002). Dietary management of inborn errors of metabolism. Indian J Pediatr 69:421426.

Knerr I, Weinhold N, Vockley J \& Gibson KM (2012). Advances and challenges in the treatment of branched-chain amino/keto acid metabolic defects. J Inherit Metab Dis 35:29-40.

Kolacek S, Grguric J, Percl M \& Booth IW (1996). Home-made modular diet versus semielemental formula in the treatment of chronic diarrhoea of infancy: a prospective randomized trial. Eur J Pediatr 155:997-1001.

Leonard JV \& Morris AA (2000). Inborn errors of metabolism around time of birth. Lancet 356:583-587.

Manoli I \& Venditti CP (2016). Disorders of branched chain amino acid metabolism. Transl Sci Rare Dis 1:91-110.

Marriage B (2010). Nutrition management of patients with inherited disorders of branchedchain amino acid metabolism in P Acosta (ed). Nutrition management of patients with inherited metabolic disorders (pp. 175-236) Jones and Bartlett Publishers, Sudbury, MA.

Martin-Hernandez E, Lee PJ, Micciche A, Grunewald S \& Lachmann RH (2009). Longterm needs of adult patients with organic acidaemias: outcome and prognostic factors. $J$ Inherit Metab Dis 32:523-533.

Saudubray JM, Nassogne MC, de Lonlay P \& Touati G (2002). Clinical approach to inherited metabolic disorders in neonates: an overview. Semin Neonatol 7:3-15. 
Strauss KA, Wardley B, Robinson D, Hendrickson C, Rider NL, Puffenberger EG, Shellmer D, Moser AB \& Morton DH (2010). Classical maple syrup urine disease and brain development: principles of management and formula design. Mol Genet Metab 99:333-345.

Vockley J \& Ensenauer R (2006). Isovaleric acidemia: new aspects of genetic and phenotypic heterogeneity. Am J Med Genet C Semin Med Genet 142C:95-103.

Wappner R \& Gibson K (2006). Disorders of leucine metabolism. in N Blau, G Hoffmann, J Leonard and J Clarke (eds). Physician's guide to the treatment and follow-up of metabolic diseases (pp. 59-79) Springer-Verlag, Heidenlberg.

World Health Organization (2006). WHO child growth standards: length/height-for-age, weight-for-age, weight-for-length, weight-forheight and body mass index-for-age: methods and development. From https://www.who.int/ publications/i/item/924154693X [Retrieved July 1 2020].
Yannicelli S (2006). Nutrition therapy of organic acidaemias with amino acid-based formulas: emphasis on methylmalonic and propionic acidaemia. J Inherit Metab Dis 29:281-287.

Zand DJ, Brown KM, Lichter-Konecki U, Campbell JK, Salehi V \& Chamberlain JM (2008). Effectiveness of a clinical pathway for the emergency treatment of patients with inborn errors of metabolism. Pediatrics 122:11911195. 